\title{
Lingual Schwannoma: A Case Report
}

\author{
Manish Munjal ${ }^{1}$, Archana Arora ${ }^{1 *}$, Damanpreet Singh ${ }^{1}$, Gopika Talwar ${ }^{1}$ and Japsimran Nagpal ${ }^{2}$ \\ ${ }^{1}$ Deptt of ENTHNS, Dayanand Medical college and Hospital, Ludhiana, Punjab, India \\ ${ }^{2}$ Dayanand Medical college and Hospital, Ludhiana, Punjab, India
}

\begin{abstract}
Schwannomas are benign nerve sheath tumors uncommonly seen in the oral cavity. The etiology is largely unknown and there is no sex predilection and can occur in a vast age group. Magnetic Resonance Imaging is the investigation of choice and transoral resection allows for removal of this tumor in a vast majority of cases. Malignant transformation in these tumors is exceedingly rare. Reported here is a $2 x 2 \mathrm{~cm}$ schwannoma on the lateral border of the tongue, in a $19 \mathrm{yr}$ old male for the last 8 months. Complete transoral excision with primary closure was carried out. Histopathological examination of the surgical specimen revealed features of schwannoma.
\end{abstract}

Keywords: Schwannoma, Neurilemmoma, Antoni A and B

\section{Introduction}

A schwannoma, or neurilemmoma, is a slow growing benign tumor of unknown etiology that arises from the Schwann cells of the neural sheath of the peripheral, cranial or autonomic nerves. There is no gender preference and occurs most commonly between the ages of 20 and 50 years. [1]

Approximately $25-45 \%$ of the Extracranial schwannomas occur in the head and neck region, with only $1 \%$ located in the mouth. The parapharyngeal space is the most common site in the head and neck region whereas when present intraorally, the tongue is the most commonly involved. [2,3]

\section{Case report}

A 19-year old male patient presented with an 8 month old swelling at the posterior part of left side of tongue. He described that the mass was small at onset and gradually became large in size. There was mild uneasiness on swallowing though not associated with any local pain nor any referred otalgia.

Intraoral 70 degree rigid telescopy revealed a firm, circumscribed globular, non tender, pinkish mass $2 \times 2 \mathrm{~cm}$ in diameter with no bleeding point or puncta, on the of the posterior part of the left side of tongue. No lymph nodes were palpable and the head and neck examination revealed no other lesions.

Contrast Enhanced Magnetic Resonance Imaging of the neck revealed a well defined polypoidal lesion which was isointense on $\mathrm{T} 1 \mathrm{w}$ and hyperintense on $\mathrm{T} 2 \mathrm{w}$; arising from the myelohyoid muscle and causing mild oropharyngeal compromise. [Fig 1]

The patient's medical history was unremarkable. Surgical complete intraoral excision was carried out using the video assisted rigid 70 degree endoscopic technique with cold instruments .a $1 \mathrm{~mm}$ border of clinically uninvolved surrounding tissue was resected. The postoperative course was uneventful. The mobility of the tongue was within normal range.

Histopathological examination of the surgical specimen showed a well circumscribed $2.5 \mathrm{X} 2 \mathrm{X} 1 \mathrm{~cm}$ tumor composed of hypercellular and hypocellular areas with no mitosis or nuclear atypia. The overlying epithelium and deep resected margins were free of tumor. These features were suggestive of benign schwannoma. [Fig 2]

The patient has been followed up for last 2 years and there has been no evidence of recurrence so far.

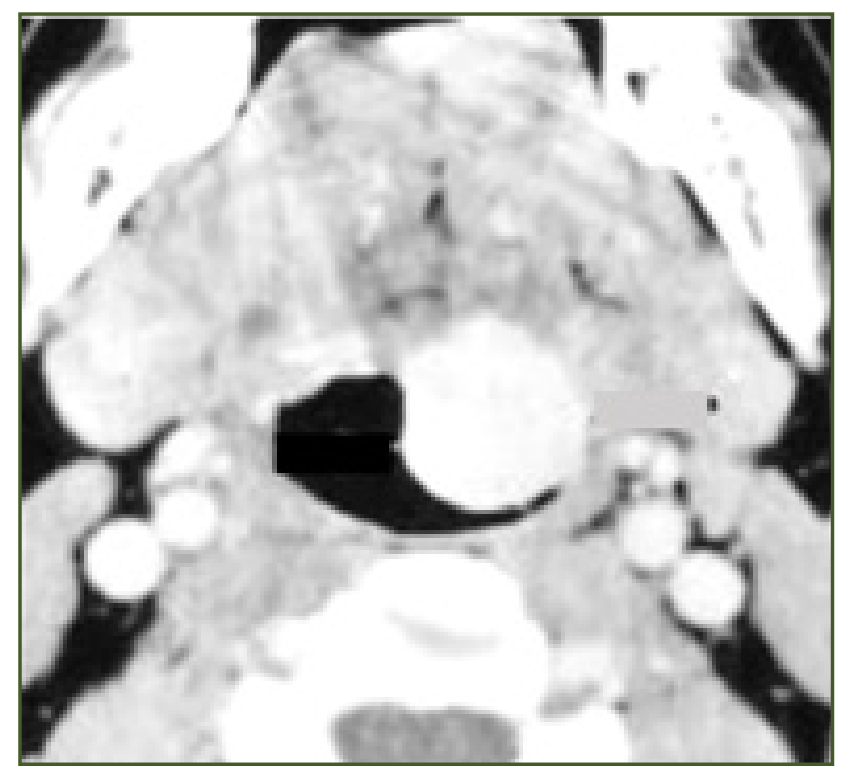

Fig. 1: Well demarcated hyper-intense lesion. 


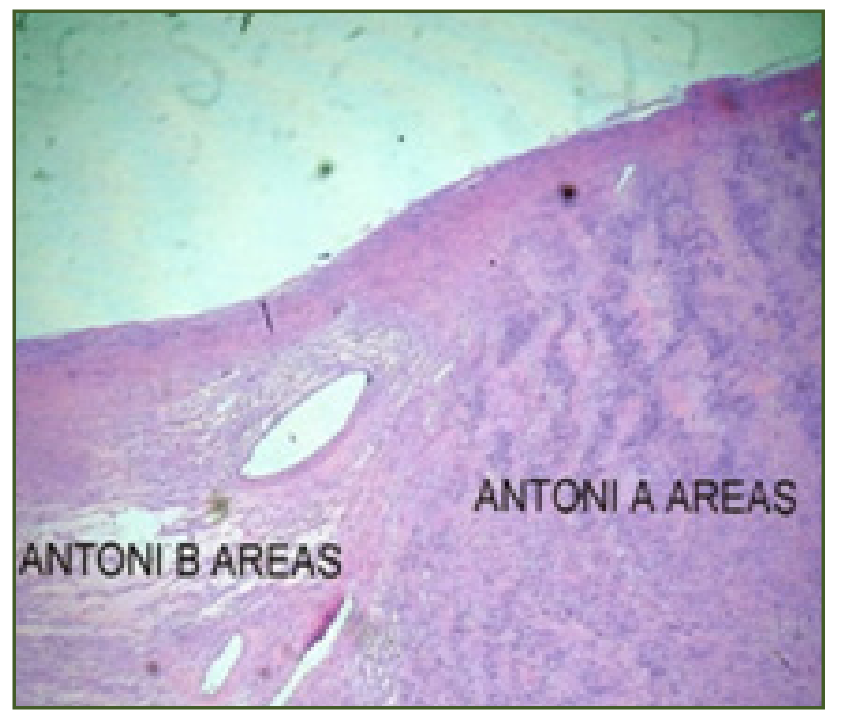

Fig. 2: Section showing Antoni A \& B areas, suggestive of Schwannoma.

\section{Discussion}

Schwannomas were first described by Verocay in 1908. [5] Schwannoma is a variable sized, slow-growing benign tumor of the nerve sheath originating from the Schwann cells and presenting usually as a solitary, well circumscribed, firm and painless lesion. It manifests as an insidiously growing mass of long duration, producing few symptoms. [2,3]

Schwannoma of the tongue mostly presents on the lingual surface without any gender preference. A schwannoma on the base of tongue is usually larger at presentation than one on the tongue itself, since it is asymptomatic initially. Complete resection is achievable and recurrence is uncommon [4].

The imaging modality of choice for schwannomas of the tongue is Magnetic Resonance Imaging (MRI) as it gives an accurate measurement and localization in relation to nearby structures. On MR, these tumors appear smooth, well demarcated, and not invading the surrounding musculature and are isointense to muscle on T1-weighted images and homogenously hyperintense on T2-weighted images.

Surgical excision is the treatment of choice with the transoral route being the most commonly employed. Other approaches like submandibular and suprahyoid pharyngotomy were used for base of tongue schwannomas that were deemed difficult to approach by the transoral route. Nowadays, the $\mathrm{CO}_{2}$ laser has also been used for such excision. A complete resection ensures no recurrence. [3]

Histologically all of these tumors are encapsulated. Inside the capsule, two main regions are identified; Antoni A and Antoni B. Antoni A regions consist of closely packed spindle-shaped Schwann cells arranged in rows around an eosinophilic area surrounded by a palisade of spindle cells. Free amorphous substance between these rows form the Verocay bodies which are oval or linear in shape. Antoni B regions are composed of fewer and loosely arranged Schwann cells that lack the organoid Verocay bodies. Immunohistochemical markers, S-100 and Leu 7, support the diagnosis further.

Differentialdiagnosisincludesneurofibromas, leiomyomas, rhabdomyomas, hemangiomas, lymphangiomas, lipomas, pyogenic granulomas, etc. [3,5]

\section{Conclusion}

Lingual schwannomas are benign slow growing tumors that are usually asymptomatic. These lesions are amenable to complete surgical excision via transoral route and have a low recurrence rate and very less chances of malignant transformation. MRI and Histopathological examination is diagnostic. The 70 degree video assisted endoscopic technique is excellent for surgical intervention and follow up examinations.

Due ethical consent was taken from the participants of the study

\section{References}

1. Lingual Schwannoma Involving the Posterior Lateral Border Of The Tongue In A Young Individual: Case Report. Pereira L J, Patrícia P. Journal of Clinical Pediatric Dentistry. September 2008, Vol. 33, No. 1, pp. 59-62

2. A rare case of schwannoma of the tongue. Grabowski L. Otolaryngologia Polska. Volume 62, Issue 2, 2008, Pages 191-4

3. Schwannoma of the tongue: two case reports and review of the literature. Cohen M, Wang M.B. European Archives of Oto-Rhino-Laryngology. November 2009, Volume 266, Issue 11, pp 1823-9

4. Schwannoma (neurilemmoma) of the tongue. Hwang C. Acta Oto-Laryngologica Vol. 126, Iss. 8,2006 Pages 861-5.

5. A Schwannoma of the Soft Palate in a Child: Histological and Immunohistochemical Features and Surgical Method. Rahpeyma A Jafarian A.Iranian Journal of Otorhinolaryngology No.2, Vol.24, Serial No.67, Spring-2012,95-9

*Corresponding author:

Dr Archana Arora, 159-C rishi nagar, Ludhiana-141001. Punjab, India

Phone: +91 9888780218

Email: Drarchana.ent@gmail.com

Financial or other Competing Interests: None.

Date of Submission : 02.04.2017

Date of Acceptance : 15.04.2017

Date of Publication : 21.04.2017 